\title{
USO DE LAS REDES SOCIALES DIGITALES PROFESIONALES Y CIENTÍFICAS: EL CASO DE LAS 3 UNIVERSIDADES GALLEGAS
}

\author{
The use of professional and scientific social networks: The \\ case of three Galician universities
}

Francisco Campos-Freire y José Rúas-Araújo

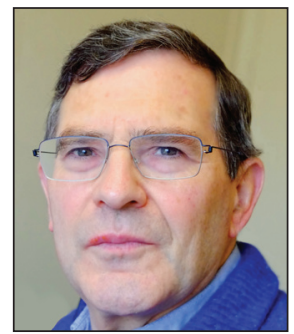

Francisco Campos-Freire es doctor por la Universidad de Santiago de Compostela (USC) y profesor de su Facultad de Ciencias de la Comunicación desde 1991. Es miembro del Grupo de Novos Medios de la USC, coordinador de la Red Internacional de Investigación de Gestión de los Medios de Comunicación (R2014/026 Xescom) e investigador del Programa Prometeo (2014-2016) del Senescyt en las Universidades Técnica Particular de Loja y Pontificia Católica de Ibarra en Ecuador. http://orcid.org/0000-0003-1831-1838

Universidad de Santiago de Compostela, Facultad de Ciencias de la Comunicación Campus Sur. Av. Castelao, s/n. 15705 Santiago de Compostela, España francisco.campos@usc.es

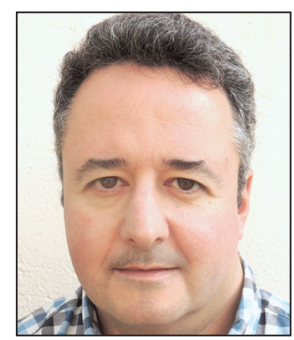

José Rúas-Araújo es profesor titular de la Facultad de Ciencias Sociales y de la Comunicación de la Universidad de Vigo y coordinador del grupo de investigación Necom: neurocomunicación, publicidad y política. Sus ámbitos de investigación son la comunicación política, electoral e institucional y las neurociencias aplicadas a las ciencias sociales y la persuasión.

http://orcid.org/0000-0002-1922-9644

Universidad de Vigo, Facultad de Ciencias Sociales y de la Comunicación Campus A Xunqueira, s/n. 36005 Pontevedra, España http://necom.uvigo.es joseruas@uvigo.es

\section{Resumen}

Una encuesta a 5.500 profesores e investigadores de las tres universidades de Galicia recibió 463 respuestas (8,42\%). Se revela el ascenso y popularidad de las redes sociales digitales profesionales y científicas entre los académicos, pero también su todavía bajo conocimiento y uso eficiente de ellas. El $90 \%$ de los docentes universitarios gallegos que respondieron se ha inscrito en alguna red profesional o académica, pero sólo un $8,64 \%$ se considera como usuario experto. Linkedln y ResearchGate son las que tienen más miembros, aunque con una utilización media-baja. Los de las áreas de ingenierías, biomédicas y ciencias naturales usan más esas dos redes citadas, mientras que en las ramas sociales y humanísticas se decantan por Academia.edu. El 32,8\% del personal docente e investigador (PDI) que respondió las usa al menos una vez al mes para difundir y seguir publicaciones, en primer lugar, y luego para conseguir más citas y reputación.

\section{Palabras clave}

Redes sociales digitales científicas; Redes profesionales; Identidad digital; Reputación digital; Metamedios; Metamedios científicos; LinkedIn; ResearchGate; Academia.edu; Investigadores; Encuestas.

\begin{abstract}
A survey conducted among 5,500 professors and researchers from the three Galician universities received 463 answers. The results reveal a rise in popularity of professional and scientific digital social networks, but it also shows a lack of knowledge about them and inefficient use of them. Ninety percent of Galician university professors are present in a professional or academic network, but only 8.64 percent consider themselves experts. LinkedIn and ResearchGate have the largest number of members, although their average use is medium or low. Biomedical, engineering, and natural sciences scholars use more networks than the two previously mentioned; and social sciences and humanities scholars prefer Academia.edu. In total, 32.8 percent of the three universities' scholars that answered the survey use social networks at least once a month. Social networks are used primarily to disseminate and follow publications, and, secondly, to get citations and improve reputation.
\end{abstract}




\section{Keywords}

Scientific social networks; Professional networks; Digital reputation; Scientific metamedia; Metamedia; LinkedIn; Researchgate; Academia.edu; Researchers; Surveys.

Campos-Freire, Francisco; Rúas-Araújo, José (2016). “Uso de las redes sociales digitales profesionales y científicas: el caso de las 3 universidades gallegas". El profesional de la información, v. 25, n. 3, pp. 431-440.

http://dx.doi.org/10.3145/epi.2016.may.13

\section{Introducción}

Las redes formales e informales -llámense asociaciones, revistas, simposios, foros, debates, escuelas o instituciones académicas superiores- han dado vida a los llamados colegios visibles e invisibles a través de los que se articulan las relaciones de la ciencia (De-Solla-Price, 1986; Crane, 1969; 1972; Wagner, 2009). Primero fueron las sociedades secretas y científicas del siglo XVII, luego las revistas, comunidades epistémicas y de prácticas (Haas, 1992; Wenger, 1998) o las comunidades virtuales online (Rheingold, 2004; ProuIx; Heine, 2009) que dieron paso a los metamedios sociales digitales (Manovich, 2005, 2008). Las herramientas y redes sociales digitales científicas son la arquitectura virtual de esos colegios invisibles o redes de citación e intercambio científico (Campos-Freire; Rivera-Rogel; Rodríguez, 2014).

Los sitios de redes sociales digitales (Boyd; Ellison, 2007; Beer, 2008) surgen de la evolución de los blogs y otras herramientas de la web 2.0, a través de ecosistemas de autocomunicación, interacción, colaboración y autoorganización (Boley; Chang, 2007) de los científicos. Son medios digitales de la web 2.0 que adoptan la arquitectura de las redes sociales reales tradicionales (Celaya, 2008; Christakis; Fowler, 2010). Pueden ser generalistas (Facebook, Twitter, YouTube), profesionales (LinkedIn) y especializados o temáticos (redes científicas). Esta arquitectura tecnocomunicativa acelera e impulsa una nueva dimensión del conocimiento llamada e-ciencia 2.0 (Nosek, 2012), más abierta, colaborativa y ciudadana (Charvolin; Micoud; Nihart, 2007; Flichy, 2010), trastocando los paradigmas e indicadores de reconocimiento de la reputación (De-Pablos-Coello; Mateos-Martín; Túñez-López, 2013) y sus modelos de negocio (CamposFreire, 2015).

Las llamadas web 1.0 y 2.0 son "colmenas de innovación disruptiva" (Christensen, 1997) que despliegan enjambres de servicios y sistemas digitales que alteran los modelos tradicionales de gestión de la comunicación e información. Las "batallas" de la información general y especializada pasaron primero de los archivos estáticos de las bibliotecas a los buscadores en red y de estos a diversas herramientas:

- gestores de referencias (EndNote, Zotero, CiteULike);

- identificadores de objetos y nombres digitales (DOI, creado en 2000 y Orcid, en 2012);

- altmétricas o nuevas métricas de los datos (Torres-Salinas; Cabezas-Clavijo; Jiménez-Contreras, 2013; ÁlvarezGarcía; Gértrudix-Barrio; Rajas-Fernández, 2014);

- redes sociales digitales generalistas (MySpace, Facebook, Flickr, YouTube, Twitter) que se desarrollan entre 1999 y 2006, y
- redes sociales digitales especializadas y temáticas (LinkedIn en 2003, Mendeley, ResearchGate y Academia.edu, todas ellas aparecidas en 2008).

Las redes sociales digitales científicas son plataformas de metamedios (medios informáticos y de comunicación evolucionados) que funcionan como ecosistemas de interacción mediante perfiles públicos o semipúblicos de afiliación ofreciendo una serie de servicios:

- difusión de las propias publicaciones y seguimiento de las de otros colegas;

- acceso abierto a millones de artículos y trabajos;

- curación de contenidos científicos;

- interconexión con constelaciones de aplicaciones de filtrado y búsquedas;

- desarrollo de conversaciones y relaciones de colaboración para investigaciones;

- aplicación de dispositivos de inteligencia artificial semántica;

- sistemas de crowdreview y socialreview;

- ofertas de empleo docentes;

- gestión de la identidad digital;

- formación de rankings e indicadores de reputación académica;

otros metaservicios digitales.

Las redes sociales digitales científicas son plataformas de metamedios que funcionan como ecosistemas de interacción mediante perfiles públicos o semipúblicos de afiliación ofreciendo una serie de servicios

Si el origen es relativamente reciente, su campo de investigación lo es más todavía, aunque ya son centenares los artículos publicados sobre ellas. Buena parte de ellos se centra en:

- su utilidad para la Academia y la investigación (Thelwall; Kousha, 2015a; Dafonte-Gómez; Míguez-González; Puentes-Rivera, 2015; Yu et al., 2016);

- confrontación de las métricas bibliográficas tradicionales y las altmétricas (Almousa, 2011; Haustein et al., 2014; Thelwall; Kousha, 2015b; Thornley et al., 2015; Nicholas et al., 2015; Ortega, 2015; Hoffman; Lutz; Meckel, 2015; - conveniencia de promover su uso eficiente (GonzálezDíaz; Iglesias-García; Codina, 2015);

- teoría de la gratificación que produce su usabilidad (Johnson; Kaye, 2015); 
- riesgos de manipulación científica de los nuevos actores robóticos posthumanos (Gourlay, 2014).

Un estudio del Consejo Superior de Investigaciones Científicas (CSIC) (Ortega, 2015), a partir de 10.000 perfiles de investigadores españoles de ocho áreas, constata diferencias entre disciplinas, con pequeña agregación o superposición en más de una red y poca relación entre indicadores bibliométricos tradicionales y nuevas altmétricas.

Otro trabajo sobre 100 investigadores de cuatro países y disciplinas diferentes (Nicholas et al., 2015) concluye que la presencia en las redes todavía es pasiva, que sus indicadores de reputación sólo registran papers y textos de conferencias y que aún es pronto para su evaluación concluyente.

Una encuesta sobre el uso de ResearchGate y Academia.edu (Corvello; Felicetti, 2014) les atribuye confianza interprofesional, autoeficiencia, ventajas esperadas y adquisición de conocimiento y reconocimiento. $Y$ en otra encuesta sobre la valoración de redes sociales, la mitad de los académicos reconocen que afectan a su actividad profesional, situando en primer lugar a LinkedIn (68\%), seguida de Academia.edu, Mendeley y ResearchGate (Haustein et al., 2014).

La popularidad de las redes digitales entre los científicos crece exponencialmente de año en año. La presencia de miembros de las 24 universidades más prestigiosas del Reino Unido aumentó entre 2012 y 2013 en las dos principales redes digitales mundiales, ResearchGate y Academia.edu, en un $14,5 \%$ y $82,7 \%$ respectivamente (Campos-Freire; Rivera-Rogel; Rodríguez, 2014).

Las redes digitales cambian la dinámica de la comunicación científica (Thelwall; Kousha, 2015a) acelerando el ciclo de retroalimentación del conocimiento, duplicando el acceso abierto a las investigaciones y reduciendo a más de la mitad el tiempo de retorno de las citas (Madisch, 2015). Un estudio de Academia.edu sobre 34.940 artículos científicos sostiene que los artículos agregados a esa red tienen un $41 \%$ más de citas tras el primer año, un $50 \%$ al trienio y un $73 \%$ después del quinto año (Niyazov et al., 2015).

La penetración en las 77 universidades españolas alcanzaba en 2015 una media del 50\%, aunque en algunas de ellas no llegaba al 10\% (González-Díaz; Iglesias-García; Codina, 2015). Según este mismo estudio, sólo dos de las univer-

Tabla 1. Distribución de las respuestas en los siete campus de las tres universidades gallegas

\begin{tabular}{|l|l|c|c|c|c|}
\cline { 3 - 6 } \multicolumn{2}{c|}{} & Frecuencia & Porcentaje & $\begin{array}{c}\text { Porcentaje } \\
\text { válido }\end{array}$ & $\begin{array}{c}\text { Porcentaje } \\
\text { acumulado }\end{array}$ \\
\hline \multirow{4}{*}{ Válidos } & Santiago & 134 & 28,9 & 28,9 & 28,9 \\
\cline { 2 - 6 } & Coruña & 112 & 24,2 & 24,2 & 53,1 \\
\cline { 2 - 6 } & Vigo & 108 & 23,3 & 23,3 & 76,5 \\
\cline { 2 - 6 } & Ourense & 35 & 7,6 & 7,6 & 84,0 \\
\cline { 2 - 6 } & Lugo & 31 & 6,7 & 6,7 & 90,7 \\
\cline { 2 - 6 } & Pontevedra & 30 & 6,5 & 6,5 & 97,2 \\
\cline { 2 - 6 } & Ferrol & 13 & 2,8 & 2,8 & 100,0 \\
\hline & Total & 463 & 100,0 & 100,0 & \\
\hline
\end{tabular}

sidades gallegas (Santiago y Vigo) destacaban entre las 20 instituciones académicas españolas con mayor presencia en ResearchGate y Academia.edu (en el puesto 11 y 20, por una parte, y 13 y 20 por otra, respectivamente). Los resultados moderadamente positivos de A Coruña deben mejorarse, como acreditan Fernández-Marcial y González-Solar (2015) en otra investigación sobre el uso de las herramientas de gestión de la identidad digital a través de Orcid, ResearcherID, Scopus, Google Scholar, ResearchGate y Mendeley.

En diciembre de 2015 las tres universidades públicas gallegas tenían 5.277 miembros en ResearchGate, el 95,98\% de su plantel docente.

- La Universidad de Santiago de Compostela, que ocupa el puesto 127 del ranking de ResearchGate entre las universidades europeas, tenía agregados 2.522 miembros, 14.265 publicaciones, un $R G$ Score del 27,911.97 y un impacto de 38,370.97;

- La Universidad de Vigo, en el puesto 245 de ese ranking europeo, agrega 1.511 miembros, 6.250 publicaciones, un $R G$ Score de 16,018.79 y un impacto de 14,516.90;

- La Universidade da Coruña, en el puesto 365, agrega 1.244 miembros, 3.089 publicaciones, un RG Score de 10,058.79 y un impacto de 14,516.46.

El $R G$ Score es una altmétrica de popularidad, visibilidad e interacción individual del perfil productivo de los investigadores que, a través de agregación, forma el ranking de las respectivas universidades. El ranking de impacto es la suma de las métricas tradicionales de las publicaciones.

\section{Objeto y metodología}

Se presenta una encuesta que forma parte de una investigación más amplia para conocer la penetración real, uso, popularidad, valoración e impacto de las redes digitales científicas. Es el primer trabajo de este tipo que se realiza en España, aplicado directamente a la recepción, tomando como estudio de caso las tres universidades gallegas; porque pese a que la popularidad de las redes es alta, poco se sabe de su uso real (Hoffman; Lutz; Meckel, 2015). La hipótesis de base -abordada también en otras publicaciones analíticas recientes- es que las redes profesionales y científicas son populares pero poco conocidas y usadas en todas sus posibilidades.

Para la realización de la encuesta se repartió un cuestionario online (mediante Google Forms) al conjunto de los investigadores y profesores de las tres universidades gallegas, mediante las listas de distribución interna de correo electrónico, y de los servidores y administradores de las cuentas y listados del PDI de cada universidad, tras cursar la correspondiente solicitud a los respectivos responsables y garantizar tanto la inclusión de los datos e identidad de los promotores de la investigación como el tratamiento anónimo de las respuestas obtenidas. El cuestionario constaba de 27 preguntas, con un tiempo estimado de respuesta de ocho minutos. La encuesta se realizó y procesó en dos etapas, entre marzo y junio, y entre octubre y noviembre de 2015. 
Tabla 2. Uso de las redes sociales profesionales y académicas

\begin{tabular}{|c|c|c|c|c|c|c|c|}
\hline & \multicolumn{2}{|c|}{$\mathbf{N}$} & \multirow{2}{*}{ Media } & \multirow{2}{*}{ Mediana } & \multirow{2}{*}{$\begin{array}{c}\text { Desviación } \\
\text { típica }\end{array}$} & \multirow{2}{*}{ Mínimo } & \multirow{2}{*}{$\begin{array}{l}\text { Máximo } \\
\text { válidos }\end{array}$} \\
\hline & Válidos & Perdidos & & & & & \\
\hline Linkedln & 418 & 45 & 1,53 & 1,00 & 1,476 & 0 & 5 \\
\hline Academia.edu & 335 & 128 & 1,18 & 0,00 & 1,630 & 0 & 5 \\
\hline ResearchGate & 394 & 69 & 2,12 & 2,00 & 1,727 & 0 & 5 \\
\hline Scilink & 260 & 203 & 0,06 & 0,00 & 0,417 & 0 & 5 \\
\hline Nature Network & 260 & 203 & 0,03 & 0,00 & 0,296 & 0 & 3 \\
\hline Otra & 245 & 218 & 0,58 & 0,00 & 1,454 & 0 & 5 \\
\hline
\end{tabular}

El universo de estudio fue el personal docente e investigador (PDI), compuesto por funcionarios (catedráticos y profesores titulares), personal fijo y contratado laboral de las tres universidades gallegas, correspondiente a los dos campus de la USC (ciudades de Santiago de Compostela y Lugo), tres de la UVigo (ciudades de Vigo, Ourense y Pontevedra) y dos de la UdC (ciudades de A Coruña y Ferrol), distribuido del siguiente modo $^{1}$ :

- 2.099 docentes e investigadores de la USC (1.664 del campus de Santiago y 435 del campus de Lugo);

- 1.983 de la UVigo (1.270 del campus de Vigo, 414 del campus de Ourense y 299 del campus de Pontevedra);

- 1.416 de la UdC (1.169 del campus de A Coruña y 247 del campus de Ferrol).

En suma, 5.498 docentes e investigadores de las tres universidades gallegas.

El total de las respuestas obtenidas fue de 463 personas $(8,42 \%)$, distribuidas del siguiente modo:

- Universidad de Vigo, 173 respuestas (37,4\%);

- Universidad de Santiago de Compostela, 165 respuestas (35,6\%);

- Universidad de A Coruña, 125 respuestas (27\%).

La distribución de las respuestas por campus se muestra en la tabla 1.

Con estos resultados, el margen de error obtenido por la aleatoriedad de la muestra es inferior al 5\%, con una precisión global del $4,328 \%$, teniendo en cuenta el número total de profesores e investigadores de todas las universidades gallegas y suponiendo que en la peor situación la probabilidad de respuesta en algún ítem de la encuesta sea de un $50 \%$, con un intervalo de confianza del $95 \%$.

\section{Resultados}

\subsection{Quiénes utilizan las redes sociales académicas}

A continuación se muestran los datos obtenidos en las tres universidades gallegas y su distribución por edad y sexo. La edad media de los que respondieron la encuesta es de 46,58 años $( \pm 9,66) y$, mayoritariamente, fueron hombres (55,3\%).

Por lo que respecta a la procedencia del PDI por ámbitos y áreas de conocimiento, se agruparon siguiendo los criterios de distribución por campos dispuestos por la Comisión Nacional Evaluadora de la Actividad Investigadora (Cneai) ${ }^{2}$. Las ramas en las que se registró un mayor número de respuestas fueron:

- matemáticas, física, química, biología y ciencias de la naturaleza, con un total de 102 respuestas (22,0\%);

- ingenierías, con 97 respuestas (21,0\%);

- arte y humanidades, historia y expresión artística, filología y lingüística, con un total de 69 respuestas $(14,9 \%)$.

El área de menor participación fue filosofía, con tan sólo dos respuestas $(0,4 \%)$.

Por categorías los profesores titulares fueron los que más respondieron, con un total de 204 respuestas obtenidas $(44,1 \%)$, seguidos de:

- contratados doctores: 87 respuestas (18,8\%);

- catedráticos de universidad: 49 respuestas (10,6\%);

- investigadores predoctorales: 41 respuestas $(8,9 \%)$;

- profesores asociados: 39 respuestas $(8,4 \%)$;

- ayudantes doctores: 19 respuestas (4,1\%);

- investigadores postdoctorales: 16 respuestas (3,5\%);

- profesores ayudantes no doctores: 8 respuestas (1,7\%).

\subsection{Cuántos las utilizan y cuáles}

Casi el 90\% (concretamente el 88,98\%) de los que respondieron admite que está presente en alguna red, profesional o académica, y tan sólo 51 de los 463 profesores e investigadores que contestaron (11,02\%), señalan que no están vinculados a ninguna.

A pesar del alto porcentaje anterior, al preguntar sobre el nivel de conocimiento y manejo de las redes académicas, la mayoría $(60,69 \%)$, reconoce que su manejo es muy bási-
Tabla 3. Frecuencia y porcentaje de uso de ResearchGate

\begin{tabular}{|l|l|r|r|r|r|}
\cline { 3 - 6 } \multicolumn{2}{c|}{} & Frecuencia & $\%$ & $\begin{array}{c}\text { Porcentaje } \\
\text { válido }\end{array}$ & $\begin{array}{c}\text { Porcentaje } \\
\text { acumulado }\end{array}$ \\
\hline \multirow{5}{*}{} & 0 & 110 & 23,8 & 27,9 & 27,9 \\
\cline { 2 - 6 } & 1 & 51 & 11,0 & 12,9 & 40,9 \\
\cline { 2 - 6 } & 2 & 55 & 11,9 & 14,0 & 54,8 \\
\cline { 2 - 7 } & 3 & 85 & 18,4 & 21,6 & 76,4 \\
\cline { 2 - 7 } & 4 & 47 & 10,2 & 11,9 & 88,3 \\
\cline { 2 - 7 } & 5 & 46 & 9,9 & 11,7 & 100,0 \\
\cline { 2 - 7 } & Total & 394 & 85,1 & 100,0 & \\
\hline Perdidos & Sistema & 69 & 14,9 & & \\
\hline Total & 463 & 100,0 & & & \\
\hline
\end{tabular}


Tabla 4. Valoración de contenidos y utilidad de las redes

\begin{tabular}{|c|c|c|c|c|c|c|c|}
\hline & \multicolumn{2}{|c|}{$\mathbf{N}$} & \multirow{2}{*}{ Media } & \multirow{2}{*}{ Mediana } & \multirow{2}{*}{$\begin{array}{c}\text { Desviación } \\
\text { típica }\end{array}$} & \multirow{2}{*}{ Mínimo } & \multirow{2}{*}{ Máximo } \\
\hline & Válidos & Perdidos & & & & & \\
\hline Linkedln & 343 & 120 & 2,59 & 3,00 & 1,303 & 0 & 5 \\
\hline Academia.edu & 186 & 277 & 3,01 & 3,00 & 1,577 & 0 & 5 \\
\hline ResearchGate & 302 & 161 & 3,41 & 4,00 & 1,363 & 0 & 5 \\
\hline Scilink & 53 & 410 & 1,09 & 0,00 & 1,510 & 0 & 5 \\
\hline Nature Network & 53 & 410 & 1,25 & 0,00 & 1,709 & 0 & 5 \\
\hline
\end{tabular}

co, concretamente para hacer ajustes mínimos, compartir documentos o introducir algunos méritos, y el porcentaje de quienes indican que apenas saben nada o desconocen su funcionamiento es del $29,38 \%$ (16,58\% señalan que su conocimiento es mínimo y $12,53 \%$ que lo desconocen totalmente). Tan sólo un $8,64 \%$ se consideran usuarios expertos.

Las redes digitales cambian la dinámica de la comunicación científica acelerando el ciclo de retroalimentación del conocimiento

Entre quienes están agregados a alguna red, el mayor porcentaje se encuentra en la de carácter profesional, Linkedln $(67,17 \%)^{3}$, seguida de las académicas ResearchGate $(61,34 \%)$ y Academia.edu (30,67\%), muy por delante de otras como Scilink (1,30\%), Nature Network (0,43\%), la red social del profesorado gallego Redeiras, promovida por la Xunta de Galicia, con solamente tres personas agregadas, o BiomedExpert, Frontiers y Mendeley, estas últimas con un único profesor o investigador que indica estar vinculado en cada caso.

Cuestión aparte es el uso que los profesores e investigadores apuntan sobre estas redes, cuya utilización señalan, en la mayoría de los casos, como media-baja, siendo las más usadas ${ }^{4}$, ResearchGate, con una media de 2,12 ( $\pm 1,73)$, seguida a bastante distancia de LinkedIn, con una media de $1,53( \pm 1,48)$ y Academia. edu, con una media de 1,18 $( \pm 1,64)$.

Desde el punto de vista de contenidos y de su utilidad, ResearchGate es la red mejor valorada por los encuestados, con una mediana de 4, seguida de Linkedln y Academia.edu, con una mediana de 3 , cada una de ellas.

El $55,5 \%$ de los encuestados (257) considera fácil el manejo de Linkedın, porcentaje que en ResearchGate es el 43,2\% (200 personas), mientras que el $44,3 \%$ de los consultados alega desconocer el manejo de Academia.edu.

A continuación se quiso observar si existían diferencias en el uso de las redes entre los docentes e investigadores por categorías profesionales, a través de la utilización de tablas de contingencia, encontrándose sólo diferencias estadísticamente significativas en el caso de ResearchGate $(p<0,001)$. En esta herramienta destaca el interés suscitado entre profesores titulares y catedráticos y, por el contrario, su escasa utilización entre profesores asociados y ayudantes no doctores, es decir, entre el personal con menores perspectivas de consolidación docente e investigadora. Los resultados se muestran en el gráfico 1.

En LinkedIn no se observaron diferencias estadísticamente significativas, lo que da muestras de su utilización por igual entre las distintas categorías del PDI universitario gallego.

Otro de los propósitos de esta investigación fue observar si existían diferencias por áreas de conocimiento en el conjunto del PDI universitario gallego, para lo cual se aplicaron tablas de contingencia, encontrándose diferencias estadísticamente significativas en Linkedln $(p=0,003)$, ResearchGate $(p<0,001)$ y Academia.edu $(p<0,001)$.

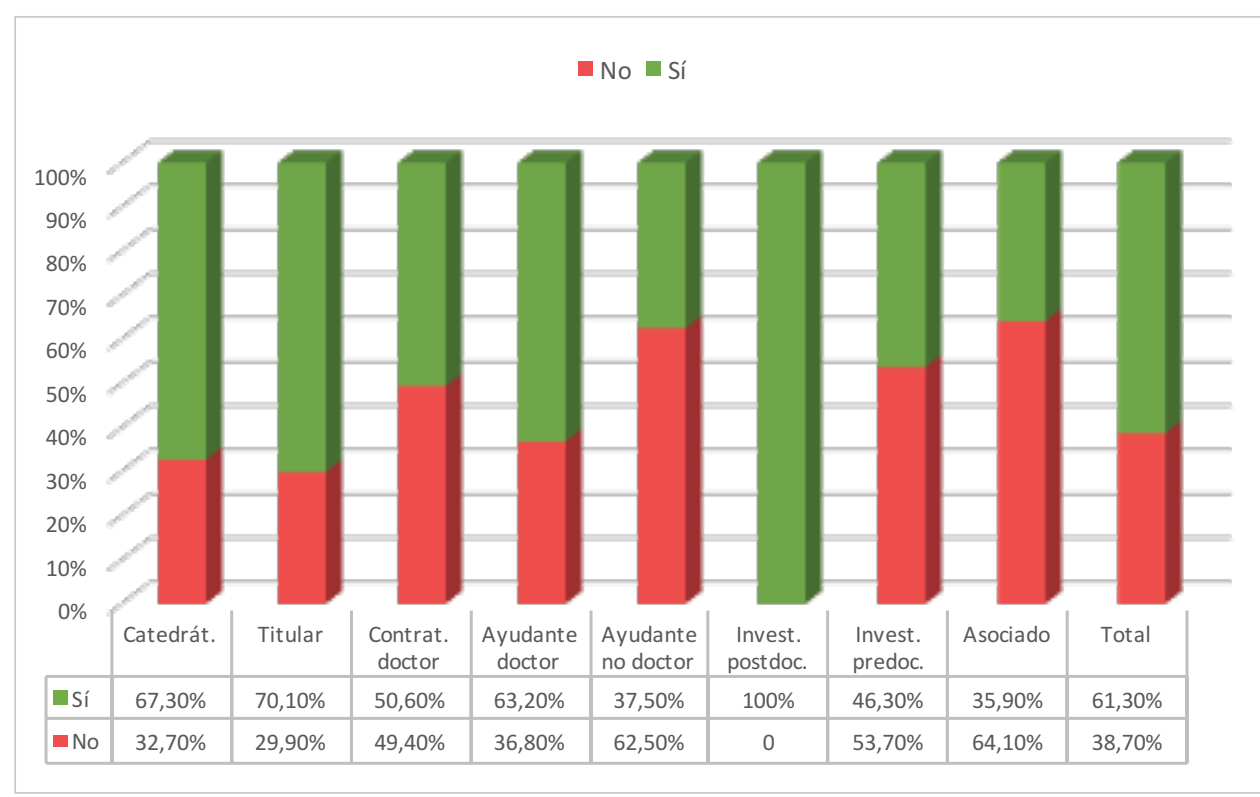

Gráfico 1. Utilización de ResearchGate por categorías profesionales 


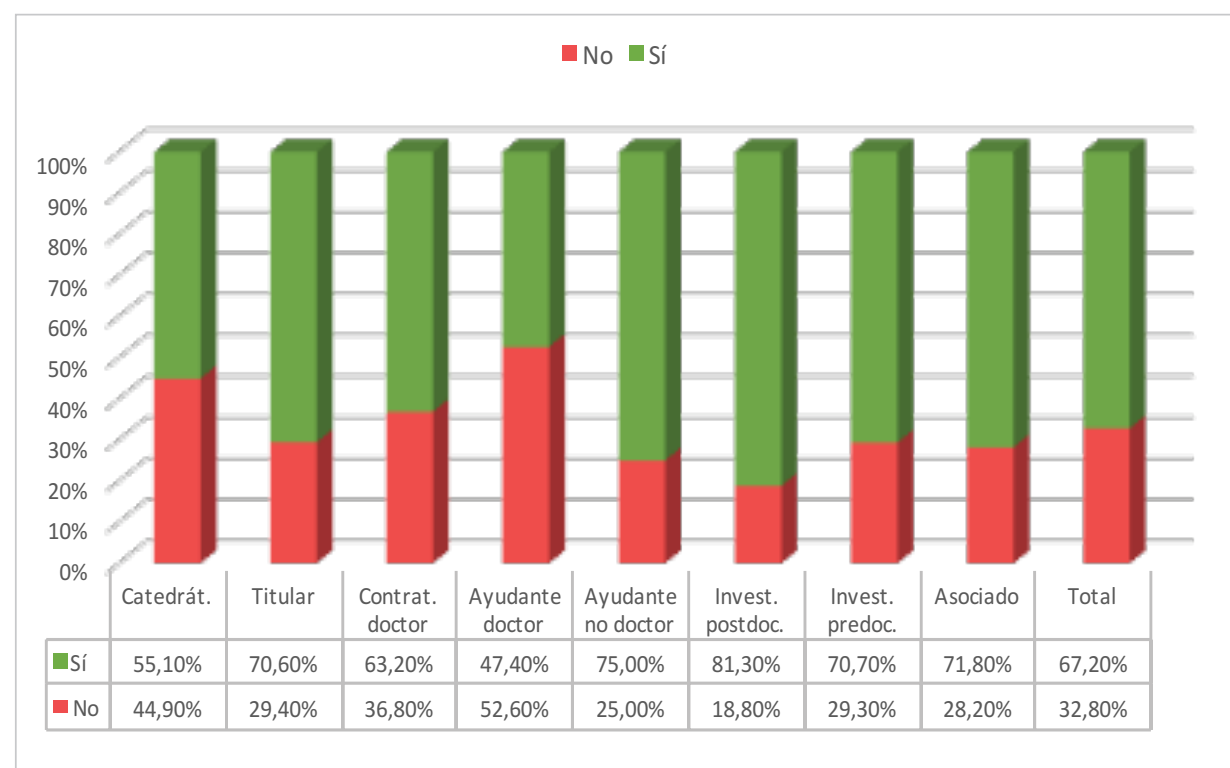

Gráfico 2. Utilización de Linkedln por categorías profesionales

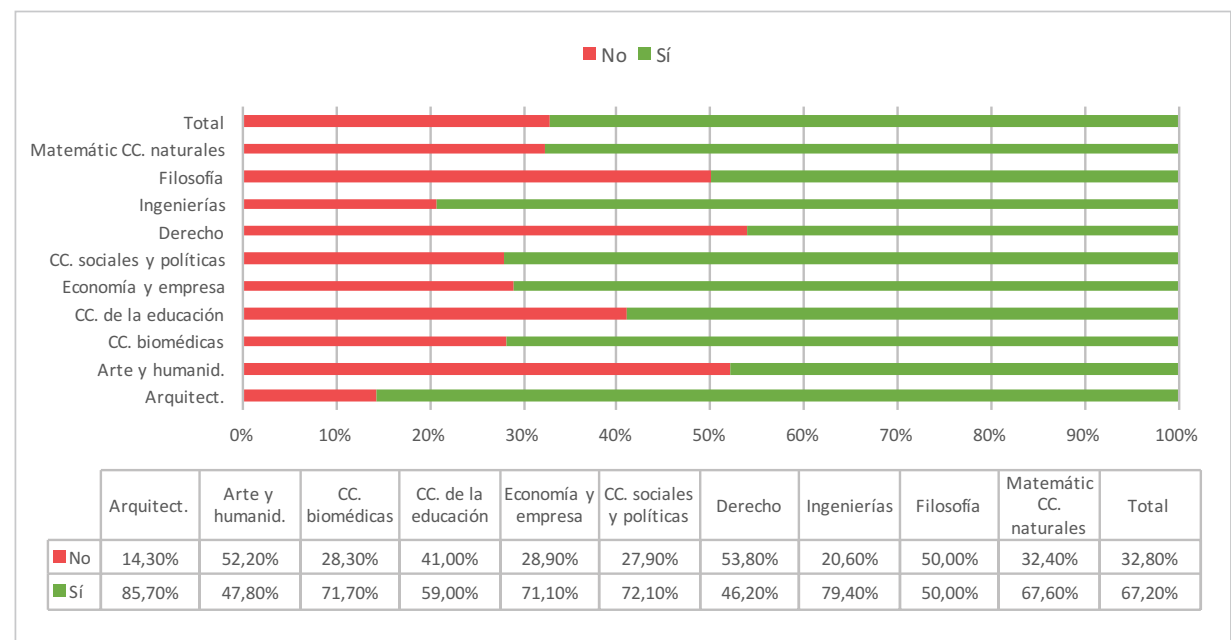

Gráfico 3. Utilización de LinkedIn por áreas y ámbitos de conocimiento

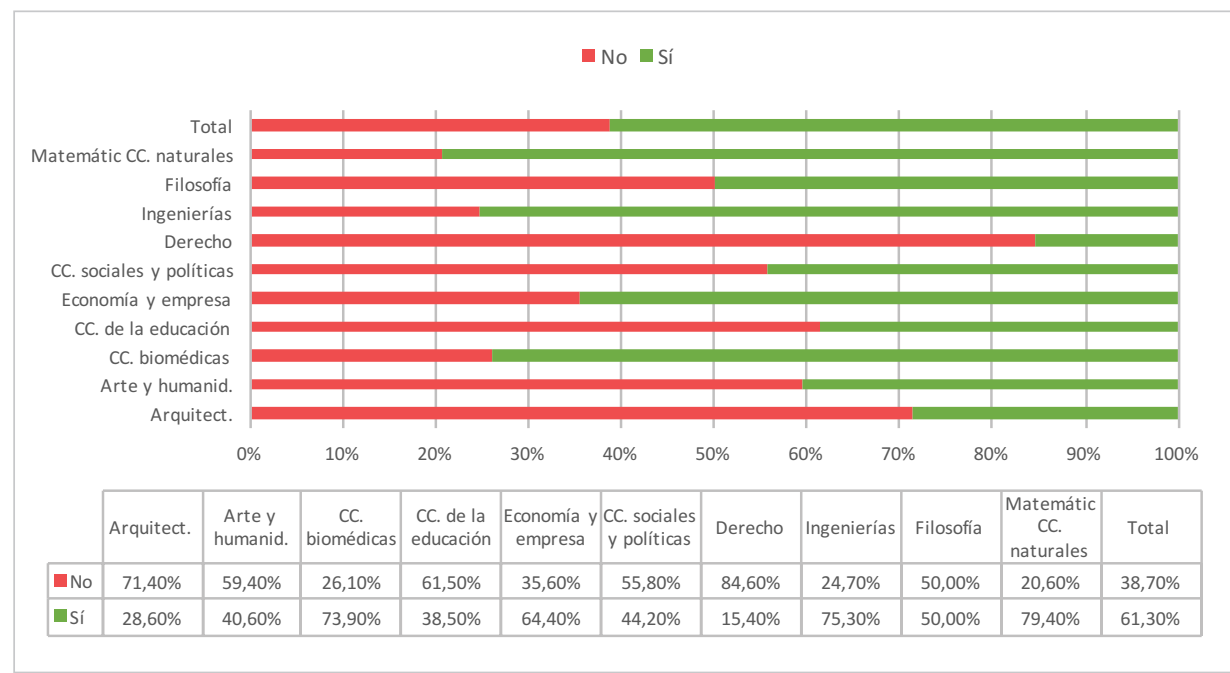

Gráfico 4. Utilización de ResearchGate por áreas y ámbitos de conocimiento
LinkedIn es más empleada por los docentes e investigadores de ámbito técnico (sobre todo en arquitectura, 85,7\%, e ingenierías, 79,4\%), frente a los vinculados a las áreas de derecho, arte y humanidades. En cualquier caso, la mayoría de los ámbitos de conocimiento presentan resultados favorables, iguales o superiores al $60 \%$ (gráfico 3).

En las redes académicas se observan diferencias bastante significativas entre ResearchGate y Academia. edu. ResearchGate registra una marcada presencia en matemáticas, física, química, biología, ciencias de la naturaleza $(79,4 \%)$, ingenierías $(75,3 \%)$ y ciencias biomédicas (73,9\%), y Academia.edu está mucho más presente en artes y humanidades $(75,4 \%)$, en contraste con el $89,1 \%$ de los docentes e investigadores de las ciencias biomédicas y el $85,6 \%$ de las ingenierías que no la utilizan. Los resultados de ambas redes académicas se muestran en los gráficos 4 y 5 .

\subsection{Para qué y con qué fre- cuencia las utilizan}

El uso más habitual de las redes académicas es conocer las publicaciones de otros investigadores y dar a conocer las propias, y también, seguir a otros investigadores o ser seguido por ellos. En un segundo nivel de importancia, se sitúa el hecho de conseguir más citas y más reputación como profesionales universitarios.

Por el contrario, son pocos los profesores e investigadores gallegos que declaran utilizar estas redes para establecer nuevos contactos, buscar nuevas ideas para sus investigaciones o ahorrar tiempo en la búsqueda de información. Aún menos todavía son los que las utilizan con afán corporativo, para promocionar sus grupos de investigación, empresas o universidades, y 
muy pocos quienes encuentran en ellas una vía para la búsqueda de trabajo, tal y como puede observarse en el gráfico 6.

Con respecto a la frecuencia de consulta o actualización, más de la mitad de los encuestados $(53,1 \%)$ reconocen que les dedican muy poco o nada de tiempo, dentro de los cuales están quienes aseguran que las consultan una vez al mes o menos $(32,8 \%)$ y quienes admiten que no le dedican tiempo (20,3\%).

\section{Conclusiones y discusión}

Los resultados de esta encuesta realizada entre docentes e investigadores de las tres universidades gallegas, con un perfil de mediana edad (46,58 años de media) y mayoritariamente hombres (55,3\% de las respuestas), muestran en líneas generales un relativo conocimiento y utilización de las redes sociales académicas y profesionales.

Estos resultados deben tomarse con cierta precaución, pues es posible que la respuesta esté sesgada y estén sobre-representados los usuarios de redes sociales. Cabría suponer que los no usuarios de redes sociales tuvieran menos interés en participar en una encuesta precisamente sobre tales redes sociales.

Casi el 90\% (88,98\%) del PDI de las tres universidades gallegas que respondió a la encuesta indica que está agregado a alguna red social, profesional o académica, pero el 60,9\% reconoce que su manejo es básico y mínimo, mientras que el porcentaje de quienes declaran que apenas saben nada o las desconocen es casi del $30 \%$. Estos datos confirman por tanto la hipótesis de partida de que las redes sociales profesionales y científicas son poco conocidas y utilizadas en todas sus posibilidades por la mayoría del PDI de las universidades gallegas.

La mayoría de usuarios están de un modo casi testimonial, sin esperar que contribuyan a incrementar las citas a sus trabajos o su reputación académica

El tiempo que les dedica el PDI universitario gallego es escaso. Más de la mitad $(53,1 \%)$ afirma que les dedican muy poco o nada de tiempo, frente al $31,9 \%$ que señalan que las consultan o actualizan regularmente.

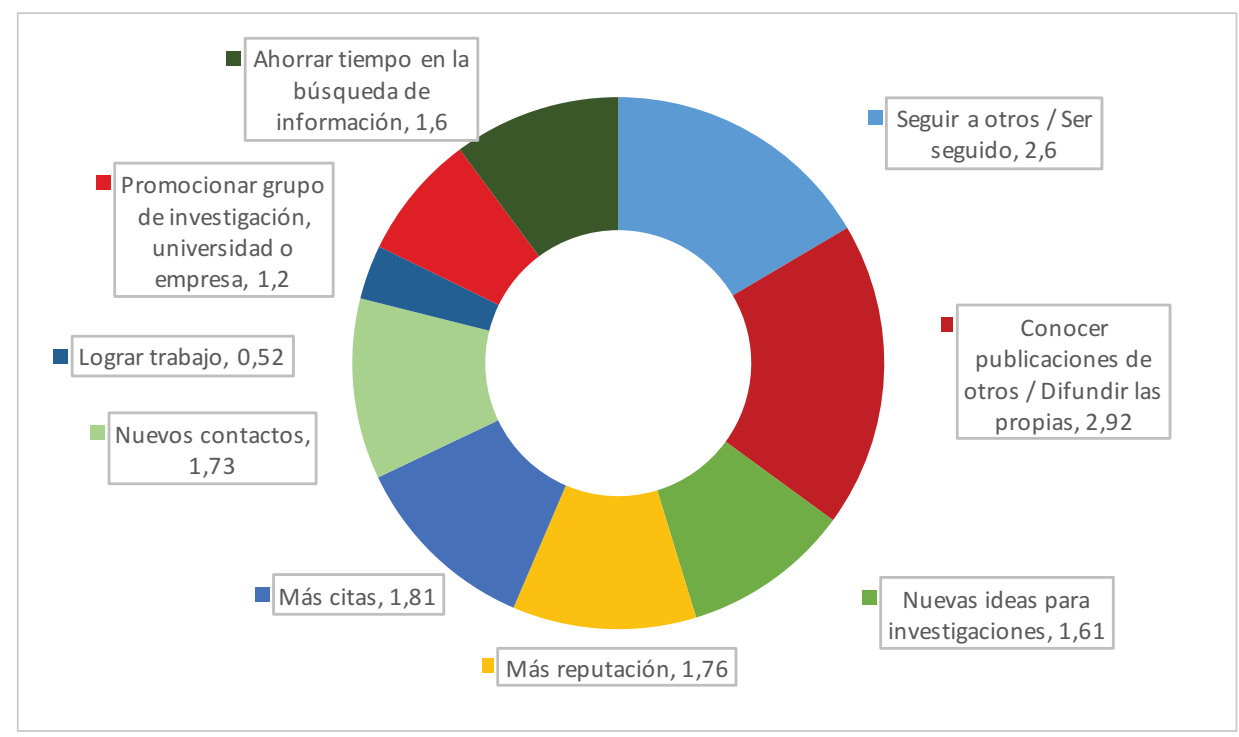

Gráfico 6. Media de uso habitual de las redes académicas
La red profesional LinkedIn es la que cuenta con un mayor porcentaje de agregados entre el PDI universitario gallego (67,17\%), seguido de las académicas ResearchGate $(61,34 \%)$ y Academia.edu (30,67\%).

En su utilización por áreas y ámbitos de conocimiento, Linkedln y ResearchGate muestran un alto porcentaje de presencia y utilización en los ámbitos científico-tecnológicos frente a áreas como arte, humanidades y derecho; todo lo contrario de lo que ocurre con Academia.edu.

Se observa cierto escepticismo en cuanto a la atención, 
valoración y utilidad de estas redes sociales, a juzgar por la baja frecuencia de visitas y actualizaciones declaradas, y también por la finalidad observada en el empleo de las mismas. La mayoría de los usuarios manifiestan cierta curiosidad por las redes sociales académicas (básicamente, para tratar de incrementar la visibilidad de sus publicaciones y seguir las de otros investigadores) e indican que están de un modo casi testimonial, sin esperar que estas redes contribuyan a incrementar las citas a sus trabajos o su reputación académica ni, mucho menos, a establecer nuevos contactos o facilitar el intercambio y la comunicación entre docentes e investigadores.

El uso más habitual de las redes académicas es conocer las publicaciones de otros investigadores, dar a conocer las propias y seguir a otros investigadores o ser seguido por ellos

La finalidad no es por tanto la búsqueda de una mayor eficacia, sino su utilización como herramientas de seguimiento y difusión, aunque con matices, en cuanto a la percepción de las mismas según las categorías profesionales. Se muestra un mayor interés por las redes académicas entre los docentes e investigadores consolidados frente a los no consolidados, mientras que, en el caso de la red profesional LinkedIn, el interés por estar presente y activo es similar en ambos casos.

Por otra parte, en la encuesta realizada también se ofrecía la posibilidad a los encuestados de dejar algún comentario, sugerencia o crítica. Entre ellos están los comentarios de quienes, habiendo respondido en la encuesta que están en otras redes distintas de las indicadas, señalan otras específicas de sus respectivos ámbitos de investigación, como SciFinder (química), MathSciNet (matemáticas), PsycINFO (psicología), RePEc (Economía), de gestores bibliográficos como RefWorks o, incluso de Web of Science.

El caso de Web of Science daría ciertamente para la inclusión de varias cuestiones al respecto, al tratarse de un metabuscador cuya indagación permitiría analizar el conocimiento y utilización de este tipo de servidores, disponibles en las universidades. Y en el caso de áreas de conocimiento, se podría observar su empleo de herramientas más específicas de cada ámbito, ampliando las opciones de respuesta y cruzando los datos obtenidos. Son algunas cuestiones de las que tomamos nota para su planteamiento en lo sucesivo, sin perder de vista la exigencia de no incrementar el tiempo de respuesta al cuestionario, que en este caso rondaba ya los ocho minutos, para lo cual será necesario incorporar nuevas cuestiones y prescindir de las observadas como menos significativas a raíz de las respuestas obtenidas.

A pesar de ello, creemos que este trabajo abre un camino para continuar y comparar la evolución de los resultados en el tiempo, tanto en lo que respecta a los docentes e investigadores, como a las herramientas que fueron objeto de estudio, desde el punto de vista cuantitativo y cualitativo.

\section{Notas}

1. Datos correspondientes al curso 2014-2015, facilitados por los servicios administrativos de las tres universidades gallegas.

2. Publicados en el BOE de 1 de diciembre de 2014, núm. 290, páginas 98204 a 98219.

3. En la encuesta y resultados obtenidos, con respecto a las redes de carácter profesional, tan sólo se menciona el caso de Linkedın, pues la utilización declarada por los encuestados de otras, como Xing o Viadeo, fue residual, con uno o dos docentes e investigadores agregados, sobre el conjunto del PDI universitario gallego.

4. En una escala de 0 a 5, donde 0 es la puntuación más baja y 5 la más alta.

Hay mayor interés por ResearchGate y Academia.edu entre los docentes e investigadores consolidados que entre los no consolidados, mientras que el interés por Linkedln es similar

\section{Créditos y financiación}

La encuesta y este artículo forman parte de las investigaciones de la Red Internacional de Investigación de la Gestión de la Comunicación (R2014/026 Xescom), apoyada en régimen competitivo por la Consellería de Cultura, Educación y Ordenación Universitaria de la Xunta de Galicia, en colaboración con un proyecto del Programa Prometeo del Senescyt de Ecuador, en la Universidad Técnica Particular de Loja (UTPL) y en la Pontificia Universidad Católica de Ibarra (Pucesi). La red Xescom está formada por cuatro grupos de investigación de las tres universidades gallegas (Novos Medios, Necom, iMarka y MILE) y otros diez de Portugal, Brasil, México, Ecuador y Colombia.

\section{Bibliografía}

Almousa, Omar (2011). "Users classification and usagepattern identification in academic social networks". En: Conference on applied electrical engineering and computing technologies (Aeect), 2011 IEEE Jordan. IEEE Conference Publications, pp. 1-6.

http://dx.doi.org/10.1109/AEECT.2011.6132525

Álvarez-García, Sergio; Gértrudix-Barrio, Manuel; RajasFernández, Mario (2014). "La construcción colaborativa de bancos de datos abiertos como instrumento de empoderamiento ciudadano". Revista latina de comunicación social, n. 69, pp. 661-683.

http://dx.doi.org/10.4185/RLCS-2014-1029

Beer, David (2008). "Social network(ing) sites... revisiting the story so far: A response to Danah Boyd \& Nicole Ellison". Journal of computer-mediated communication, v. 13, pp. 516-529.

http://dx.doi.org/10.1111/j.1083-6101.2008.00408.x

Boley, Harold; Chang, Eon K. (2007). “Digital ecosystems: 
Principles and semantics". Inaugural IEEE Intl conf on digital ecosystems and technologies, Australia.

http://citeseerx.ist.psu.edu/viewdoc/download?doi=10.1.1. 90.4199\&rep $=$ rep $1 \&$ type $=p d f$

Boyd, Danah M.; Ellison, Nicole B. (2007). "Social network sites: Definition, history, and scholarship". Journal of computer-mediated communication, v. 13, n. 1, pp. 210-230. http://dx.doi.org/10.1111/j.1083-6101.2007.00393.x

Campos-Freire, Francisco (2015). "Los sitios de redes sociales como paradigma del ecosistema digital”. En: CamposFreire, Francisco; Rúas-Araujo, José (eds.). Las redes sociales digitales en el ecosistema mediático. Cuadernos artesanos de comunicación. Sociedad Latina de Comunicación Social, pp. 13-36. ISBN: 9788416458233

http://www.cuadernosartesanos.org/abscac92.html

http://dx.doi.org/10.4185/cac92

Campos-Freire, Francisco; Rivera-Rogel, Diana; Rodríguez, Claudia (2014). "La presencia e impacto de las universidades de los países andinos en las redes sociales digitales". Revista latina de comunicación social, n. 69, pp. 571-592. http://dx.doi.org/10.4185/RLCS-2014-1025

Celaya, Javier (2008). La empresa en la web 2.0. Barcelona: Gestión 2000. ISBN: 9788498751734

Charvolin, Florian; Micoud, André; Nyhart, Lynn K. (coords.) (2007). Des sciences citoyennes? Paris: La Tour d'Aigues, Éditions de l'Aube. ISBN: 9782111281554

Christakis, Nicholas A.; Fowler, James H. (2010). Conectados. El sorprendente poder de las redes sociales y cómo nos afectan. Madrid: Taurus. ISBN: 9788430606887

Christensen, Clayton M. (1997). The innovator's dilemma: When new technologies cause great firms to fail. Harvard Business Press. ISBN: 9780875845852

http://www.hbs.edu/faculty/Pages/item.aspx?num=46

Corvello, Vicenzo; Felicetti, Alberto-Michele (2014). "Factors affecting the utilization of knowledge acquired by researchers from scientific social networks: An emprical analysis". Knowledge management: An international journal, v. 13, n. 3, pp. 15-26.

Crane, Diane (1969). "Social structure in a group of scientists: A test of the 'invisible college' hypothesis". American sociological review, v. 34, n. 3, pp. 335-352.

Crane, Diane (1972). Invisible colleges: Diffusion of knowledge in scientific communities. Chicago: University of Chicago Press. ISBN: 0226118576

Dafonte-Gómez, Alberto; Míguez-González, María-Isabel; Puentes-Rivera, Iván (2015). "Academic social networks: Presence and activity in Academia.edu and ResearchGate of communication researchers of the Galician universities". En: Rocha, Alvaro; Dias, Gançalo-Paiva; Martins, Arnaldo; Reis, Luis-Paulo; Pérez-Cota, Manuel (2015). 10 $10^{\text {th }}$ Iberian conf on information systems and technologies (Cisti), Univ. Aveiro (Portugal). ISBN: 9781479983308

http://dx.doi.org/10.1109/CISTI.2015.7170594

De-Pablos-Coello, José-Manuel; Mateos-Martín, Concha;
Túñez-López, Miguel (2013). “Google cambia el paradigma de la métrica científica". Historia y comunicación social, v. 18, pp. 225-235.

http://dx.doi.org/10.5209/rev_HICS.2013.v18.44327

De-Solla-Price, Derek J. (1986). Little science, big science and beyond. Nueva York: Columbia University Press. ISBN: 0231049579

Fernández-Marcial, Viviana; González-Solar, Llarina (2015). "Promoción de la investigación e identidad digital: el caso de la Universidade da Coruña". El profesional de la información, v. 24, n. 5, pp. 656-664.

http://dx.doi.org/10.3145/epi.2015.sep.14

Flichy, Patrice (2010). Le sacre de l'amateur. Sociologie des passions ordinaires à l'ère numérique. Paris: Seuil. ISBN: 2021031446

González-Díaz, Cristina; Iglesias-García, Mar; Codina, Lluís (2015). "Presencia de las universidades españolas en las redes sociales digitales científicas: caso de los estudios de comunicación". El profesional de la información, v. 24, n. 5, pp. 640-647. http://dx.doi.org/10.3145/epi.2015.sep.12

Gourlay, Lesley (2015). "Posthuman texts: nonhuman actors, mediators and the digital university". Social semiotics, v. 25 , n. 4 , pp. $484-500$.

http://dx.doi.org/10.1080/10350330.2015.1059578

Haas, Peter M. (1992). "Introduction: Epistemic communities and international policy coordination". International organization, v. 46, n. 1, pp. 1-35.

https://www.unc.edu/ fbaum/teaching/articles/IO-1992Haas.pdf http://www.jstor.org/stable/2706951

Haustein, Stefanie; Peters, Isabella; Bar-Ilan, Judit; Priem, Jason; Shema, Hadas; Terliesner, Jens (2014). “Coverage and adoption of altmetrics sources in the bibliometric community". Scientometrics, v. 101, n. 2, pp. 1145-1163.

http://arxiv.org/abs/1304.7300

http://dx.doi.org/10.1007/s11192-013-1221-3

Hoffman, Christian-Pieter; Lutz, Christoph; Meckel, Miriam (2015). "A relational altmetric? Network centrality on ResearchGate as an indicator of scientific impact". Journal of The Association for Information Science and Technology, v. 67, n. 4, pp. 765-775.

http://dx.doi.org/10.1002/asi.23423

Johnson, Thomas J.; Kaye, Barbara K. (2015). "Reasons to believe: Influence of credibility on motivations for using social networks". Computers in human behavior, v. 50, pp. 544-555.

http://dx.doi.org/10.1016/j.chb.2015.04.002

Madisch, ljad (2015). "The pace of scientific research is picking up". Harvard business review, 3 August.

https://hbr.org/2015/08/the-pace-of-scientific-research-ispicking-up

Manovich, Lev (2005). El lenguaje de los nuevos medios de comunicación: la imagen en la era digital. Barcelona: Paidós. ISBN: 9788449317699 
https://uea1arteycomunicacion.files.wordpress.com/2013/09/ manovich-el-legunaje-de-los-nuevos-medios.pdf

Manovich, Lev (2008). Software takes command. New York: Georgetown University.

http://faculty.georgetown.edu/irvinem/theory/ManovichSoftware-Takes-Command-ebook-2008-excerpt.pdf

Nicholas, David; Herman, Eti; Jamali, Hamid; RodríguezBravo, Blanca; Boukacem-Zeghmouri, Cherifa; Dobrowolski, Tom; Pouchot, Stephanie (2015). "New ways of building, showcasing, and measuring scholarly reputation". Learned publishing, v. 28, n. 3, pp. 169-183.

http://dx.doi.org/10.1087/20150415

Niyazov, Yuri; Vogel, Carl; Price, Richard; Lund, Ben; Judd, David; Akil, Adnan; Schwartzman, Josh; Shron, Max (2015). "Open access meets discoverability: Citations to articles posted to Academia.edu". Plos one, 17 February. http://dx.doi.org/10.1371/journal.pone.0148257

Nosek, Brian A. (2012). "An open, large-scale, collaborative effort to estimate the reproducibility of psychological science". Perspectives on psychological science, v. 7, n. 6, pp. 657-660. http://dx.doi.org/10.1177/1745691612462588

Ortega, José-Luis (2015). "Relationship between altmetric and bibliometric indicators across academic social sites: The case of CSIC's members". Journal of informetrics, v. 9, n. 1, pp. 39-49. http://dx.doi.org/10.1016/j.joi.2014.11.004

Proulx, Travis; Heine, Steven J. (2009). "Connections from Kafka. Exposure to meaning threats improves implicit learning of an artificial grammar". Psychological science, v. 20, n. 9, p. 1125-1131.

http://www2.psych.ubc.ca/ heine/docs/Kafkagrammar.pdf

Rheingold, Howard (2004). Multitudes inteligentes. La próxima revolución social. Barcelona: Gedisa. ISBN: 8497840623 http://www.redalyc.org/pdf/860/86011409027.pdf

Thelwall, Mike; Kousha, Kayvan (2015a). "ResearchGate: Disseminating, communicating, and measuring scholarship?". Journal of the Association for Information Science and Technology, v. 66, n. 5, pp. 876-889. http://dx.doi.org/10.1002/asi.23236

Thelwall, Mike; Kousha, Kayvan (2015b). "Web indicators for research evaluation. Part 2: Social media metrics". El profesional de la información, v. 24, n. 5, pp. 607-620. http://dx.doi.org/10.3145/epi.2015.sep.09

Thornley, Clare; Watkinson, Anthony; Nicholas, David; Volentine, Rachel; Jamali, Hamid R.; Herman, Eti; Allard, Suzie; Levine, Lenneth J.; Tenopir, Carol (2015). "The role of trust and authority in the citation behaviour of researchers". Information research, v. 20, n. 3.

http://www.informationr.net/ir/20-3/paper677.html

Torres-Salinas, Daniel; Cabezas-Clavijo, Álvaro; JiménezContreras, Evaristo (2013). "Altmetrics: nuevos indicadores para la comunicación científica en la web 2.0". Comunicar, v. XXI, n. 41, pp. 53-60. http://dx.doi.org/10.3916/C41-2013-05

Wagner, Caroline S. (2009). The new invisible college: Science for development. Washington: Brookings Institution Press. ISBN: 9780815792130

Wenger, Etienne (1998). Communities of practice: learning, meaning, and identity. Cambridge University Press. ISBN: 9780521663632

Yu, Min-Chun; Wu, Yen-Chun-Jim; Alhalabi, Wadee; Kao, Hao-Yun; Wu, Wen-Hsiung (2016). "ResearchGate: An effective altmetric indicator for active researchers?" Computers in human behavior, v. 55, pp. 1001-1006.

http://dx.doi.org/10.1016/j.chb.2015.11.007

\section{Da visibilidad a tu trabajo depositándolo en e-LIS, el mayor repositorio internacional sobre biblioteconomía, documentación y comunicación}

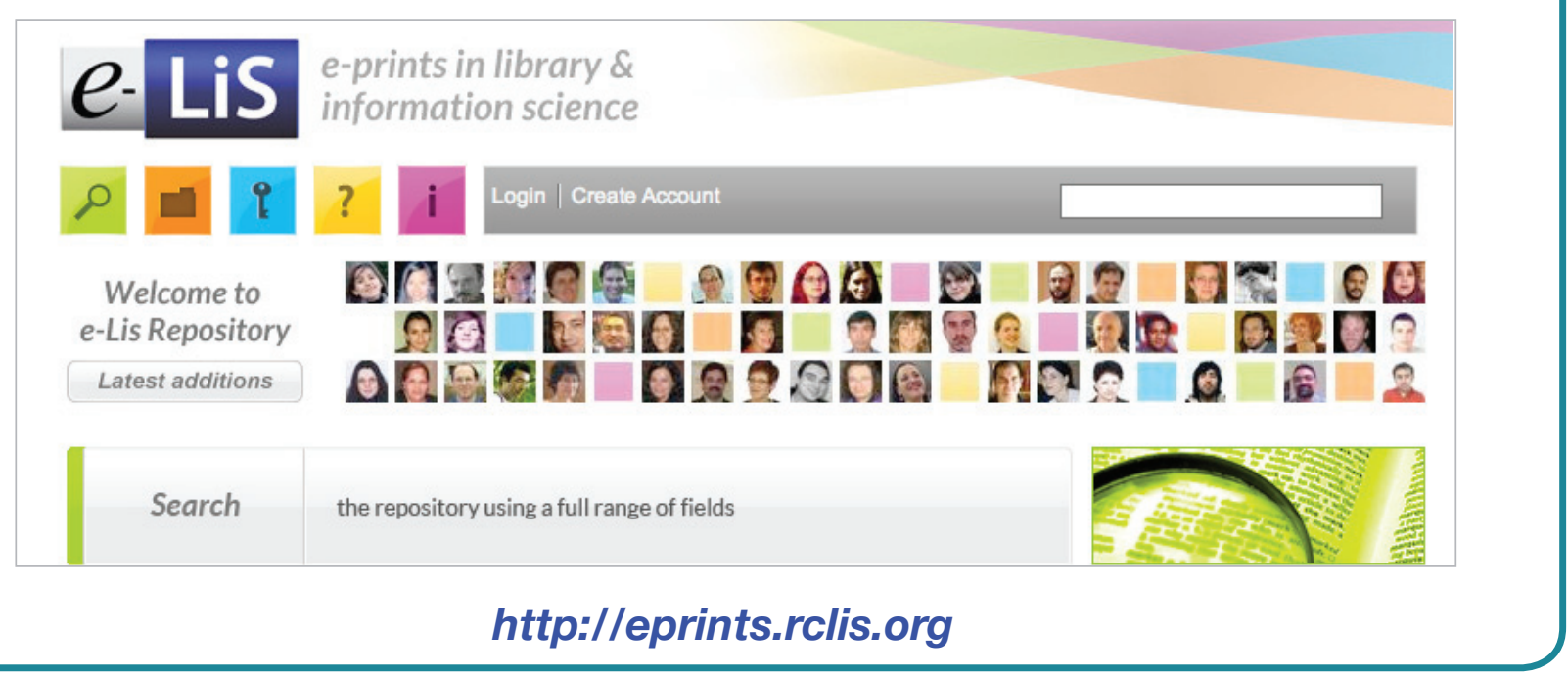

